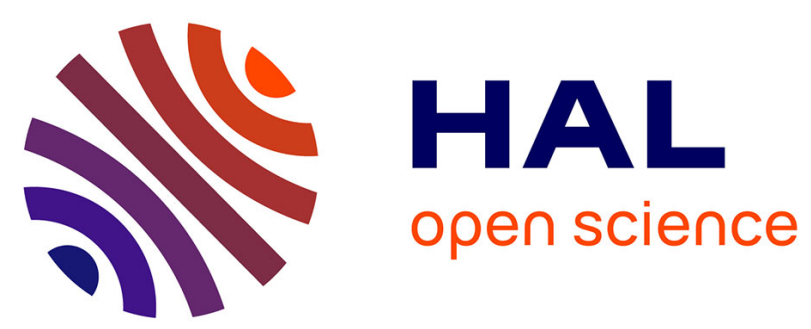

\title{
3D static and time-dependent modelling of a dc transferred arc twin torch system
}

V Colombo, E Ghedini, M Boselli, P Sanibondi, A Concetti

\section{To cite this version:}

V Colombo, E Ghedini, M Boselli, P Sanibondi, A Concetti. 3D static and time-dependent modelling of a dc transferred arc twin torch system. Journal of Physics D: Applied Physics, 2011, 44 (19), pp.194005. 10.1088/0022-3727/44/19/194005 . hal-00615137

\section{HAL Id: hal-00615137 https://hal.science/hal-00615137}

Submitted on 18 Aug 2011

HAL is a multi-disciplinary open access archive for the deposit and dissemination of scientific research documents, whether they are published or not. The documents may come from teaching and research institutions in France or abroad, or from public or private research centers.
L'archive ouverte pluridisciplinaire HAL, est destinée au dépôt et à la diffusion de documents scientifiques de niveau recherche, publiés ou non, émanant des établissements d'enseignement et de recherche français ou étrangers, des laboratoires publics ou privés. 


\title{
3D static and time-dependent modeling of a dc transferred arc twin torch system
}

\author{
V Colombo, E Ghedini, M Boselli, P Sanibondi and A Concetti \\ Department of Mechanical, Nuclear, Aviation and Metallurgical Engineering - DIEM, \\ Alma Mater Studiorum - Università di Bologna, Via Saragozza 8, 40123 Bologna, \\ Italy \\ E-mail: emanuele.ghedini@unibo.it
}

\begin{abstract}
The transferred arc plasma torch device consists of two electrodes generating a plasma arc sustained by means of an electric current flowing through the body of the discharge. Modeling works investigating of transferred electric arc discharges generated between two suspended metallic electrodes, in the so called twin torch configuration, are scarce. The discharge generated by this particular plasma source configuration is characterized by a complex shape and fluid dynamics and needs a 3D description in order to be realistically predicted. The extended discharge length that goes from the tungsten pencil cathode to the flat copper anode without any particular confinement wall and the fluid dynamics and magnetic forces acting on the arc may induce an unsteady behavior. In order to capture the dynamic behavior of a twin torch discharge, a 3D time dependent plasma arc model has been developed using a customized commercial code FLUENT form in both Local Thermodynamic Equilibrium (LTE) and non-LTE. A two temperature (2T) model has been developed taking into account only the thermal non-equilibrium effects in argon plasma. The main differences between LTE and 2T models results concern the increased extension of the horizontal section of the discharge and the predicted reduced (of about 60-80V) voltage drop between the electrodes when using a $2 \mathrm{~T}$ model.
\end{abstract}

\section{Introduction}

The transferred arc plasma torch device, in its more general configuration, consists of two electrodes generating a plasma arc sustained by means of an electric current flowing through the body of the discharge, transferring power from electric field to gas by ohmic heating. A lot of modeling work has been done in order to characterize the behavior of transferred arcs in their typical configurations, like free burning arcs or cutting plasma torches [1], but very little has been done in the investigation of transferred electric arc discharges generated between two suspended metallic electrodes in the twin torch configuration. Twin torch generated arcs differ from the other transferred arcs since the anode is not the workpiece, as shown in figure 1, while the arc may or may not pass through a bath of molten material, depending on the specific application or design. This kind of device has been used mainly in reactors for waste treatment incineration and inertization, or for metallurgical applications [2-4], while on the patent side also some other applications are claimed [5]. 

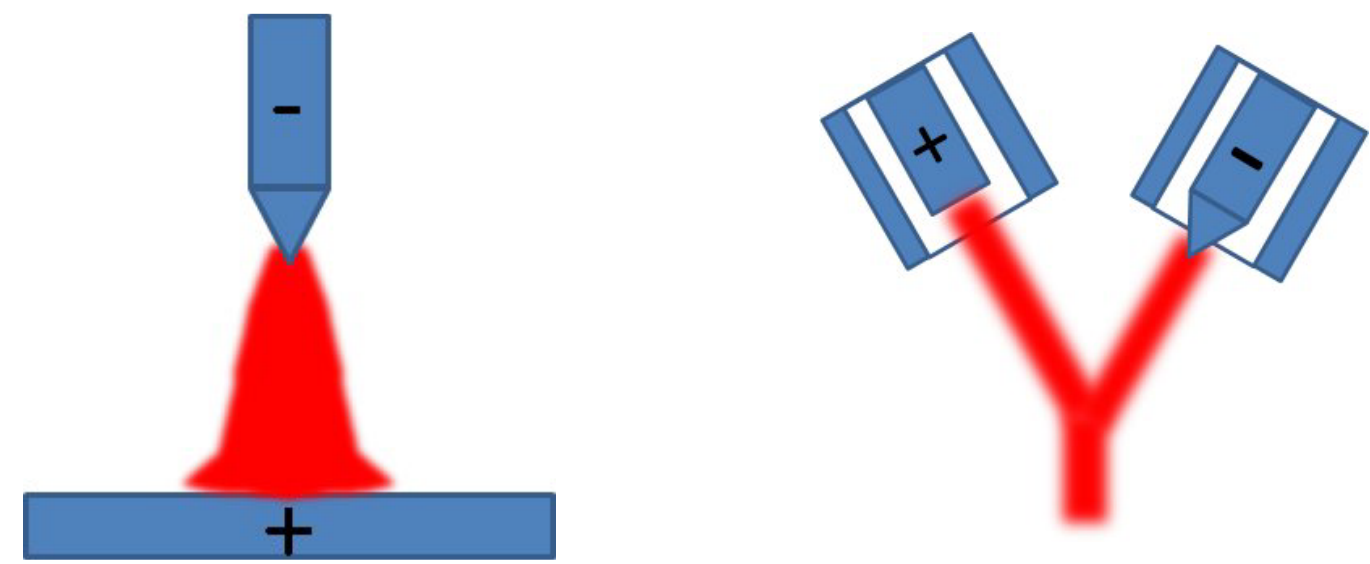

Figure 1. Schematic of a typical free burning transferred arc (left) and of a twin torch system (right).

The advantages of such device are:

- the arc can be elongated and the energy is exerted directly on the process material or on the surface of the component, the powder, gas or dispersed liquid, not in the torch;

- enhanced convection leading to a more homogeneous temperature in the reactor;

- electrodes can be protected by inert shrouding gas (e.g. Ar);

- several free degrees of freedom in the operating conditions (e.g. electrode to bath distance, inlet flows, current)

While disadvantages can be:

- possibility of instabilities at high current due to the magnetic interaction between the cathodic and anodic arc columns;

- side arcing can occur at high voltage;

- increase of design complexity with respect to simple graphite transferred electrode.

The complex shape and fluid dynamics of a twin torch discharge needs a 3D description in order to be predicted. Some research on the subject of DC transferred arc twin torch systems has been done in the past using a 3D steady state modeling of the discharge inside a plasma furnace devoted to combustion/vitrification of radioactive wastes [6] and for asbestos inertization [7]. In a recent paper [8] the same LTE steady state model previously used by the Authors in [7] together with the same model validation approach by means of free burning arc simulation comparison with literature experimental data, has been applied to a twin torch system for fine powders production. In [8] it is experimentally found that the stability of the coupling zone is mainly related to the included angle between the torches, meaning that above a critical angle a steady model can be used to predict the twin torch arcs. However the high speed frame in [8] shows a very irregular coupling zone with at least two connections between cathodic and anodic arcs that is not consistent with a steady state, and only averaged experimental voltage drop have been included. The extended discharge length that goes from the tungsten pencil cathode to the flat copper anode without any particular confinement wall and the fluid dynamics and magnetic forces acting on the arc may induce an unsteady behavior as shown in [9] for Local Thermodynamic Equilibrium (LTE) conditions. Unfortunately the results shown in [9] predict a higher voltage with respect to the values usually found experimentally, probably to due the LTE model underestimation of electrical conductivity in the cold regions. It is known that also at atmospheric pressure thermal and chemical non equilibrium may occur [10], leading to an overpopulation of electrons in cold regions, where LTE model predicts almost no ionization, and then to an increase of electrical conductivity. In a conventional transferred arc the non equilibrium effects are mainly located in the arc fringes [11] and have almost no influence on the arc voltage, limiting the necessity of $2 \mathrm{~T}$ calculations. In the twin torch configuration a non equilibrium region between the two 


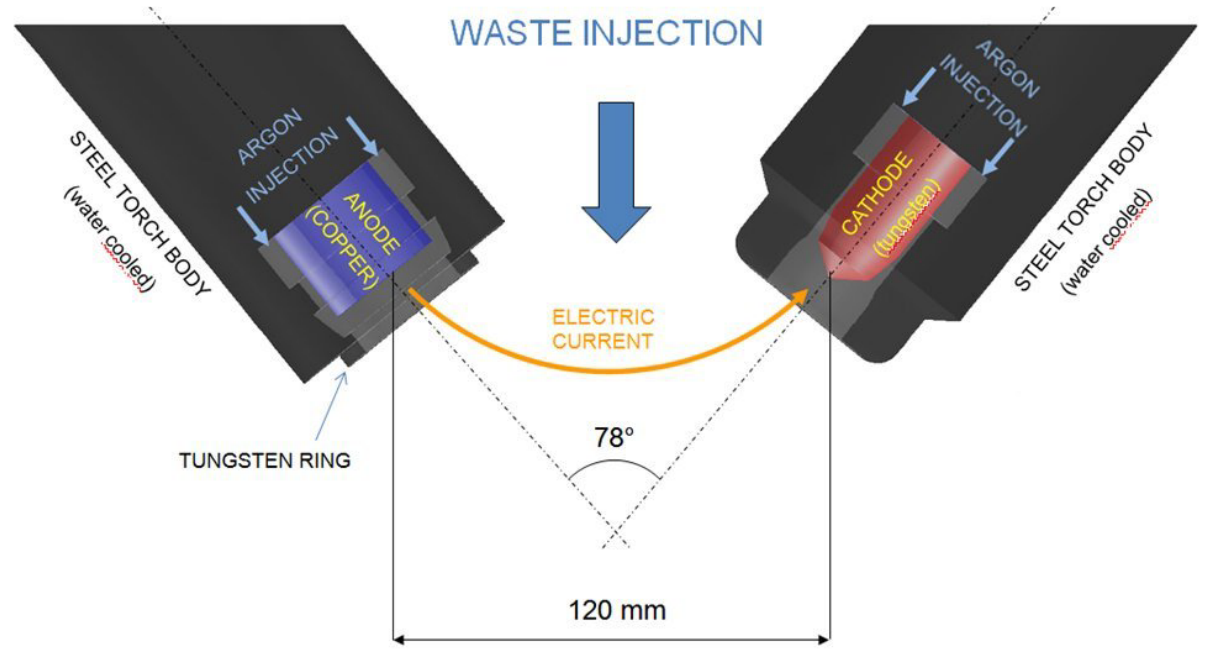

Figure 2. Schematic of the twin torch geometry.

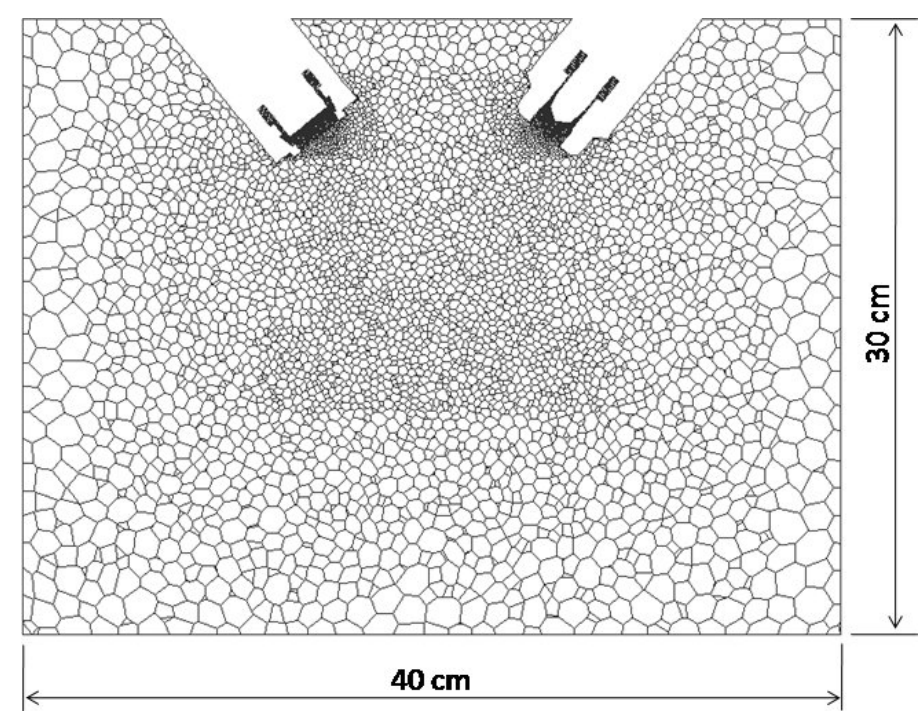

Figure 3. Detail of the mesh and computational domain.

torch would facilitate the migration of electrons from cathode to anode reducing the length of the current path and then the voltage drop. For this reasons a 3D time dependent plasma arc model including two temperature (2T) thermal non equilibrium has been developed using a customized commercial code FLUENT.

\section{Model description}

An LTE time dependent model previously developed by the Authors [7] has been extended to the non thermal equilibrium case. Turbulence effects are taken into account into the model using a $k-\varepsilon$ RNG model. Anodic and cathodic sheaths have been neglected, while electrodes interfaces are taken into account using a simplified approach, imposing a current density distribution on the cathode surface.

Neglecting sheath phenomena allows reducing the computational effort since their influence on the main body of the arcs is negligible as shown in [12]. 
In all the performed calculations, the required database of equilibrium and non equilibrium thermodynamic and transport properties has been taken from [13].

\subsection{Geometry}

The twin torch configuration is schematically represented in figure 2 and refers to a $1 \mathrm{MW}$ mobile twin torch plant dedicated to asbestos inertization from Centro Sviluppo Materiali (CSM) S.p.A, Rome, Italy [2,3]. The computational domain does not extend to the whole reactor, since the simulation of the complete $3 \mathrm{D}$ configuration would be too computationally expensive. The domain is thus limited to the region between the cathode and anode where the discharge takes place.

The computational mesh has been generated in order to cover the full volume of the discharge and it is shown in figure 3. A 700'000 polyhedral elements mesh has been generated starting from a 2 millions tetrahedrical mesh, in order to lower the computational effort and to improve numerical behavior of the code reducing the effects of badly shaped cells and model stability.

\subsection{LTE model}

The LTE model equations are summarized as follows.

The continuity and Navier-Stokes equations are

$$
\begin{gathered}
\frac{\partial \rho}{\partial t}+\nabla \cdot(\rho \vec{u})=0 \\
\frac{\partial(\rho \vec{u})}{\partial t}+\nabla \cdot(\rho \vec{u} \vec{u})=-\nabla p+\nabla \cdot \overrightarrow{\vec{\tau}}+\vec{J} \times \vec{B}+\rho \vec{g}
\end{gathered}
$$

where $\rho$ is the density, $\vec{u}$ the fluid velocity, $p$ the pressure, $\overrightarrow{\vec{\tau}}$ the viscous stress tensor and $\vec{J} \times \vec{B}$ the contribution of the Lorentz forces.

The energy conservation equation

$$
\frac{\partial(\rho h)}{\partial t}+\nabla \cdot(\rho h \vec{u})=\nabla \cdot\left(K_{e f f} \nabla T\right)+\frac{5}{2} \frac{k_{B}}{e} \vec{J} \cdot \nabla T+\vec{J} \cdot \vec{E}-Q_{\text {rad }}
$$

where $h$ is the enthalpy, $K_{\text {eff }}=K_{\text {lam }}+K_{\text {turb }}$ the effective thermal conductivity that includes the contribution of turbulence, $\vec{J} \cdot \vec{E}$ the Joule heating and $Q_{\text {rad }}$ the radiation losses calculated using a n.e.c. approach [14]. The current density has been calculated using the simplified Ohm's law, neglecting the Hall current $\vec{J}=\sigma \vec{E}$.

\section{3. $2 T$ model}

The equations for the $2 \mathrm{~T}$ thermal non equilibrium model are summarized as follows (see $[1,10]$ for details). Mass conservation and Navier-Stokes equations are the same as LTE model while separate energy equations for the species are needed. The energy equation for the heavy and electron species are

$$
\begin{gathered}
\frac{\partial \rho h_{h}}{\partial t}+\nabla \cdot\left(\rho h_{h} \vec{u}\right)=\nabla \cdot K_{h} \nabla T_{h}+Q_{E H} \\
\frac{\partial \frac{5}{2} k_{B} n_{e} T_{e}}{\partial t}+\nabla \cdot\left(\frac{5}{2} k_{B} n_{e} T_{e} \vec{u}\right)=\nabla \cdot\left(K_{e} \nabla T_{e}\right)+\frac{5}{2} \frac{k_{B}}{e} \vec{J} \cdot \nabla T_{e}+Q_{J}-Q_{r a d}-Q_{E H}
\end{gathered}
$$

where $K_{h}$ and $K_{e}$ are the thermal conductivities for heavy particles and electrons, $k_{B}$ the Boltzmann constant, $n_{e}$ the electron number density and $e$ the electron elementary charge. Electron density has been calculated using the Saha equation for $2 \mathrm{~T}$ systems assuming chemical equilibrium [10]. The 
energy exchange by collisions between electrons and heavy species $Q_{E H}$ can be expressed using a sum over all species as

$$
Q_{E H}=\sum_{s \neq e} \frac{3}{2} k_{B} \frac{2 m_{e} m_{s}}{\left(m_{e}+m_{s}\right)^{2}} v_{e s}\left(T_{e}-T_{h}\right) \delta_{e s}
$$

where $v_{e s}$ is the collision frequency between electrons and species $s$ and $\delta_{e s}$ the inelastic collision factor, that for monoatomic gas is equal to one.

The Joule heating

$$
Q_{J}=\vec{J} \cdot \vec{E}=\sigma \vec{E}_{p} \cdot\left(\vec{E}_{p}-\frac{k_{B}}{e} T_{e} \nabla \log _{n}\left(n_{e}\right)\right)
$$

is a function of the effective electric field $\vec{E}_{p}$ that is defined as

$$
\vec{E}_{p} \approx \vec{E}+\frac{\nabla p_{e}}{e n_{e}}=\vec{E}+\frac{k_{B}}{e n_{e}} \nabla n_{e} T_{e}=\vec{E}+\frac{k_{B}}{e} T_{e} \nabla \log _{n}\left(n_{e}\right)
$$

This expression can be derived from a simplified expression of the generalized Ohm's law as shown in [1].

\subsection{Electromagnetic model}

The electromagnetic field is calculated using the vector and scalar potential form of the Maxwell's equations. The vector and scalar potential equations are solved in this form

$$
\begin{gathered}
\nabla^{2} \vec{A}-\mu_{0} \sigma \vec{E}=0 \\
\nabla \cdot \sigma \nabla V_{p}=0
\end{gathered}
$$

while the effective electric field comes from the gradient of the effective scalar potential

$$
\bar{E}_{p}=-\nabla V_{p}
$$

For the LTE case the effective electric potential $V_{p}$ is assumed equal to the electric potentials $V$.

\subsection{Boundary conditions}

A parabolic profile of the current density on the cathode surface is given as a boundary condition using this relation

$$
j(r)=-j_{\max }\left[1-\left(\frac{r}{R_{0}}\right)^{2}\right]
$$

where $j_{\max }=8 \cdot 10^{8} \mathrm{~A} / \mathrm{m}^{2}$ is the maximum value of the current density on the cathode, $r$ is the distance from the axis of the cathode, $R_{0}$ is a reference value calculated imposing a given value of the total current $I=1^{\prime} 500 \mathrm{~A}$ on the cathode surface. On the anode surface the electrical potential is assumed to be zero, while on all the other surfaces of the model the current flux is assumed to be zero.

The boundary condition for the vector potential $\partial \vec{A} / \partial \hat{n}=0$ has been used on all the wall surfaces, where $\hat{n}$ is the unit vector normal to the surface. An alternative condition $\vec{A}=0$ on the walls has been used to artificially stabilize the discharge in order to define a reference steady configuration for the comparison of discharges at different operating conditions. The condition $\vec{A}=0$ avoids the fluctuations of the discharge and is to be intended not as a physically realistic condition but as a trick, that makes possible to use a steady code with a considerable saving of computational time. The temperature value given as a boundary condition on the anode and cathode comes from previous calculations developed by CSM S.p.A. and are $600 \mathrm{~K}$ and $1500 \mathrm{~K}$ respectively; however, a change in these values does not have a significant influence on the results. On all the other surfaces the temperature is fixed at $500 \mathrm{~K}$. Argon flow rate is $80 \mathrm{slpm}$ for both electrodes. 
(a) $\mathrm{t}=0 \mathrm{~ms}$
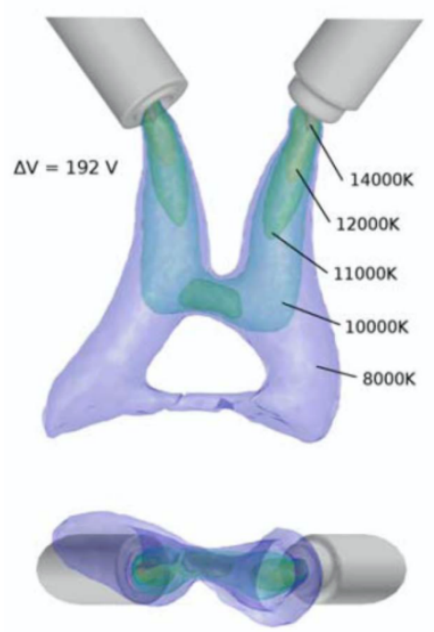

(d) $\mathrm{t}=3 \mathrm{~ms}$
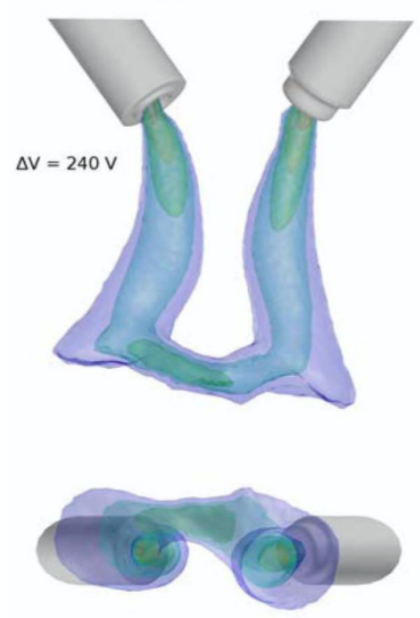

(b) $\mathrm{t}=1 \mathrm{~ms}$
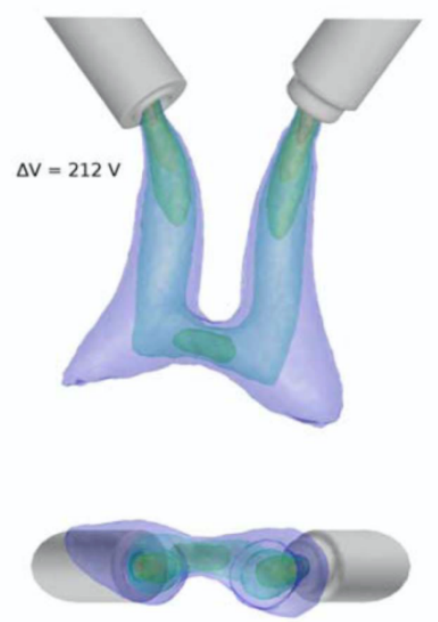

(e) $\mathrm{t}=4 \mathrm{~ms}$
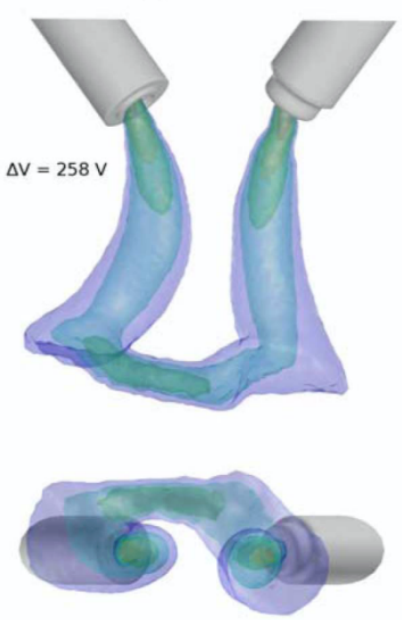

(c) $\mathrm{t}=2 \mathrm{~ms}$
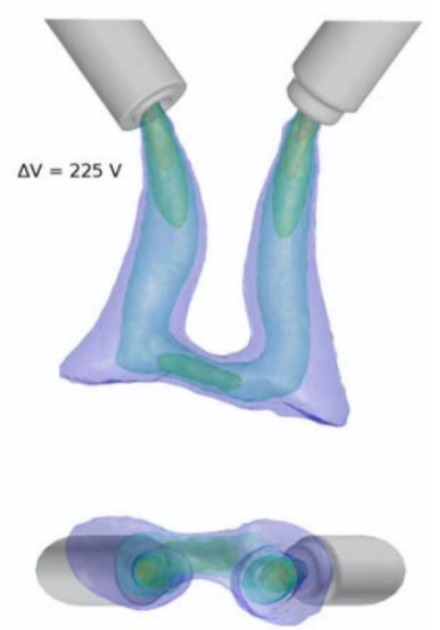

(f) $\mathrm{t}=5 \mathrm{~ms}$
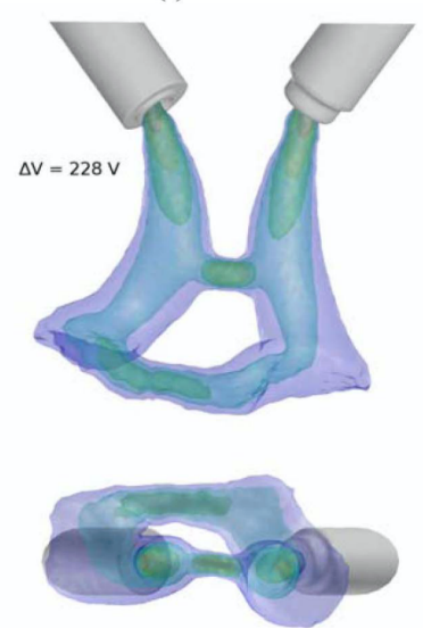

Figure 4. Side and bottom view of isotemperature surfaces with computed voltage values in the twin torch system at different time steps for $1500 \mathrm{~A}$. Isosurfaces temperature values are: $8000 \mathrm{~K}, 10000 \mathrm{~K}$, $11000 \mathrm{~K}, 12000 \mathrm{~K}$ and $14000 \mathrm{~K}$. Cathodic torch is always on the right. (from [9]). 


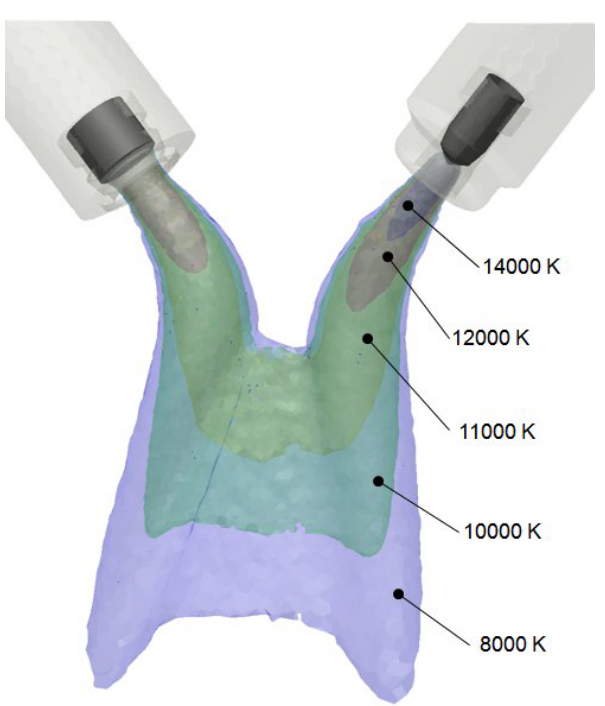

Figure 5. Isotemperature surfaces of the twin torch discharge for LTE model.
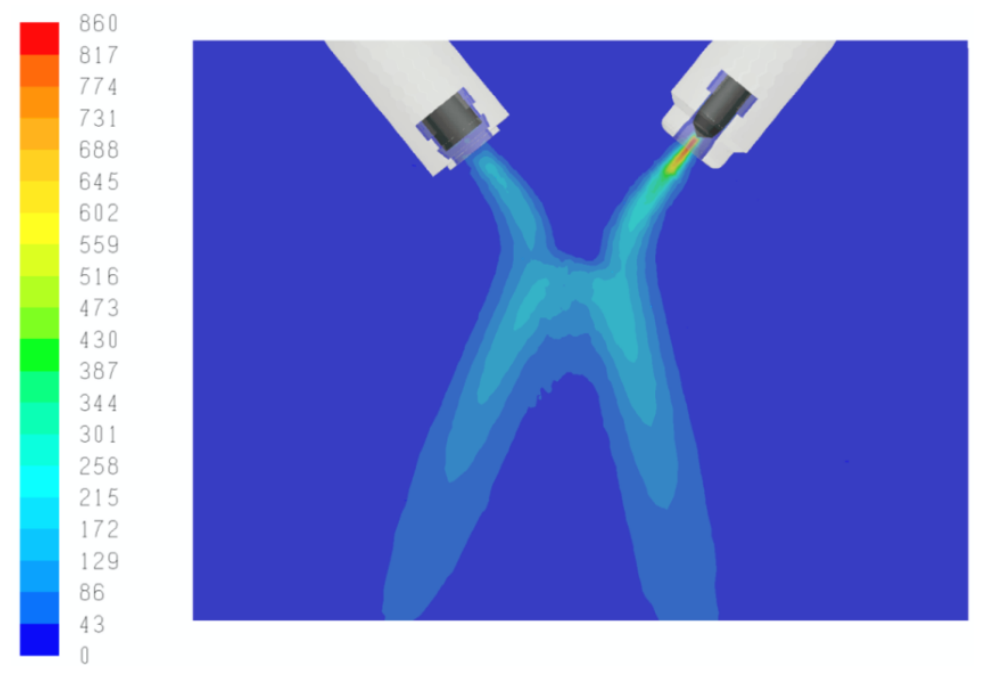

Figure 6. Velocity field $[\mathrm{m} / \mathrm{s}]$ on the plane passing through the axis of the torches for LTE model.
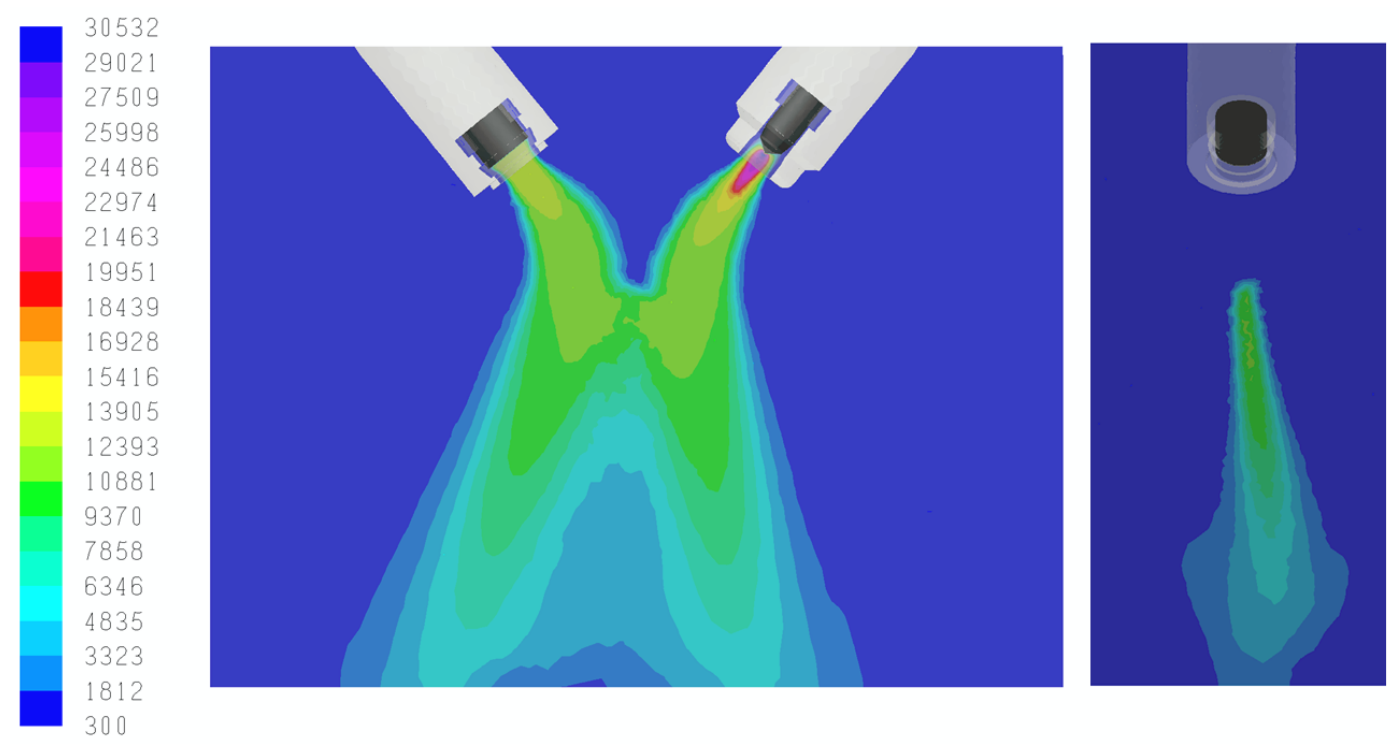

Figure 7. Front and side view of the temperature field $[\mathrm{K}]$ on the mid planes for the LTE model. 


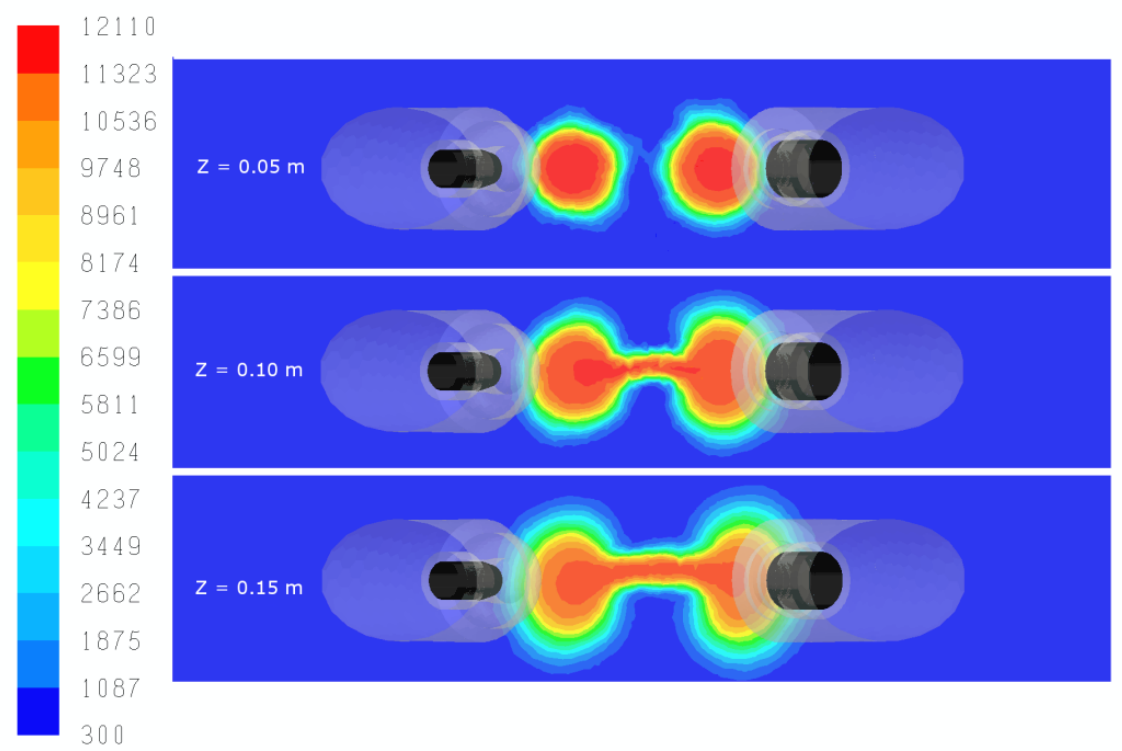

Figure 8. Temperature field $[\mathrm{K}]$ on horizontal planes for the LTE model at different vertical distance $z$ from the cathode tip.

\section{Results}

\subsection{Time dependent LTE model}

The time dependent behaviour of this device has been described in by the Authors in [8,9] and sketched in figure 4 and will only be briefly discussed here. The dynamic of the twin torch discharge involves the zone of attachment of the two plasma columns and seems to be due to an unbalancing between Lorentz and drag forces that shift downstream the attachment point, until the gradient of the electric potential creates another upstream attachment point by means of a restrike. In this way a periodic oscillation is obtained, limited to the downstream region of the discharge with only a small influence on the fields in the proximity of the electrodes.

The voltage oscillates between 190 to $260 \mathrm{~V}$ depending on the instantaneous discharge length and it is not in agreement with experimental measurements that predict values around $150 \mathrm{~V}$. This means that the LTE model tends to overestimate the discharge power. Moreover, the cathode and anode fall must be added to the model predicted voltage, and can be roughly estimated to be overall around $5-15 \mathrm{~V}$ for a tungsten cathode $[14,15]$, less then $8 \%$ of the total voltage.

\subsection{Steady state LTE model}

Since the time dependent simulation is very computational expensive, an artificial stabilization has been introduced by means of a $\vec{A}=0$ boundary condition on torch walls that prevents the motion of the zone of attachment of the two plasma columns, as explained in the previous section. Details on the discharge will be presented using the steady model. This assumption also allows easier comparison with the following $2 \mathrm{~T}$ model results, that predict a steady discharge.

In figure 5 the temperature field is shown using isosurfaces at different temperature values. It is possible to see the repulsion of the two plasma columns due to the self generated magnetic fields. This repulsion can lead to a non symmetric shape of the discharge, as shown in figure 4, that needs a 3D model to be taken into account [9]. This phenomenon is more pronounced with the increasing of the current. The velocity field is shown in figure 6 using a contour on the mid plane; the maximum velocity is about $860 \mathrm{~m} / \mathrm{s}$ and is located on the cathode tip, where the magnetic constriction is higher. In the main body of the discharge the velocity is in the order of $200 \mathrm{~m} / \mathrm{s}$. 
Figure 7 shows the details of the temperature field on two planes. The maximum plasma temperature is located near the cathode surface and is about $30^{\prime} 000 \mathrm{~K}$, while in the proximity of the anodic surface the plasma temperature reaches a value of about 13'000 $\mathrm{K}$. The main body of the discharge is at a temperature of about 11'000 K. Figure 8 shows the temperature field at different horizontal planes and the detailed shape of the horizontal connecting channel that is quite narrow with respect to the two plasma columns due to the current driven self constriction of the arc.

\subsection{Steady state $2 T$ model}

The time dependent $2 \mathrm{~T}$ model applied to the twin torch system shows no fluctuations, for both boundary conditions $\partial \vec{A} / \partial \hat{n}=0$ and $\vec{A}=0$. For this reason a steady model approach has been used also in the $2 \mathrm{~T}$ model.

Figure 9 compares the electrons and heavy particles temperatures while figures 10 and 11 show the region of non equilibrium by means of the non equilibrium parameter $\vartheta=T_{e} / T_{h}$. The highest value of $\vartheta$ is located in the region between the two plasma columns where the electric field is higher. In this region the electric field exchange energy with electrons and the collision term $Q_{E H}$ is not sufficiently high to raise the heavy species particle temperature; also because of the flow convection that pushes cold gas downward that keeps heavy particles cool. Thermal non equilibrium is also located near the walls and in the fringes of the discharge where the temperature gradients are higher.

The strong non equilibrium in the region between the two torches leads to a local increase of the electrical conductivity that facilitates the electrons migration from cathode to anode reducing the total arc length. This has an effect on the electrodes voltage as shown in figure 12 where steady LTE model predicts a voltage of $184 \mathrm{~V}$ while the $2 \mathrm{~T}$ model a voltage of $104 \mathrm{~V}$. A cathode and anode fall overall of about $5-15 \mathrm{~V}$ must be added to these values. The total voltage predicted by the $2 \mathrm{~T}$ model is the $85 \%$ of the experimental reference value of $150 \mathrm{~V}$. This underestimation could be due to the chemical equilibrium assumption, since the region between the two torches is strongly affected by flow convection that could influence the presence of the ionized species and electrons. Still, even though this underestimation, the agreement of the $2 \mathrm{~T}$ model is better than the LTE model.

The absence of fluctuations, unlike the LTE model, could be mainly related to the enhanced electrical conductivity non equilibrium region that provides a stable channel for the electrons and that is less affected by the flow drag than the LTE case.

These results show that thermal non equilibrium in arc discharges at atmospheric pressure can have an important influence on the predicted arc properties.

\section{Conclusions}

Simulations have been presented for a twin torch system using LTE and 2T approach for the prediction of the discharge properties. A method for artificially stabilize the discharge by changing the magnetic field boundary conditions has been introduced.

While LTE model predicts a fluctuation of the discharge, the $2 \mathrm{~T}$ model shows no dynamic behavior probably due to the increased electrical conductivity in the cold region between the plasma arc columns. 2T model predicts a substantial lower voltage between electrodes with respect to LTE showing that for this kind of device the LTE approach may fail in the voltage and power prediction of the plasma discharge.

Following these evidences, it is possible that chemical non equilibrium and electron diffusion, which will be introduced as a future development in the model, will have a non negligible effect on the predicted discharge properties. Parameters like electrodes distance or flow rate should be investigated in the future in order to evaluate their influence on the restrike mode and on the voltage. 


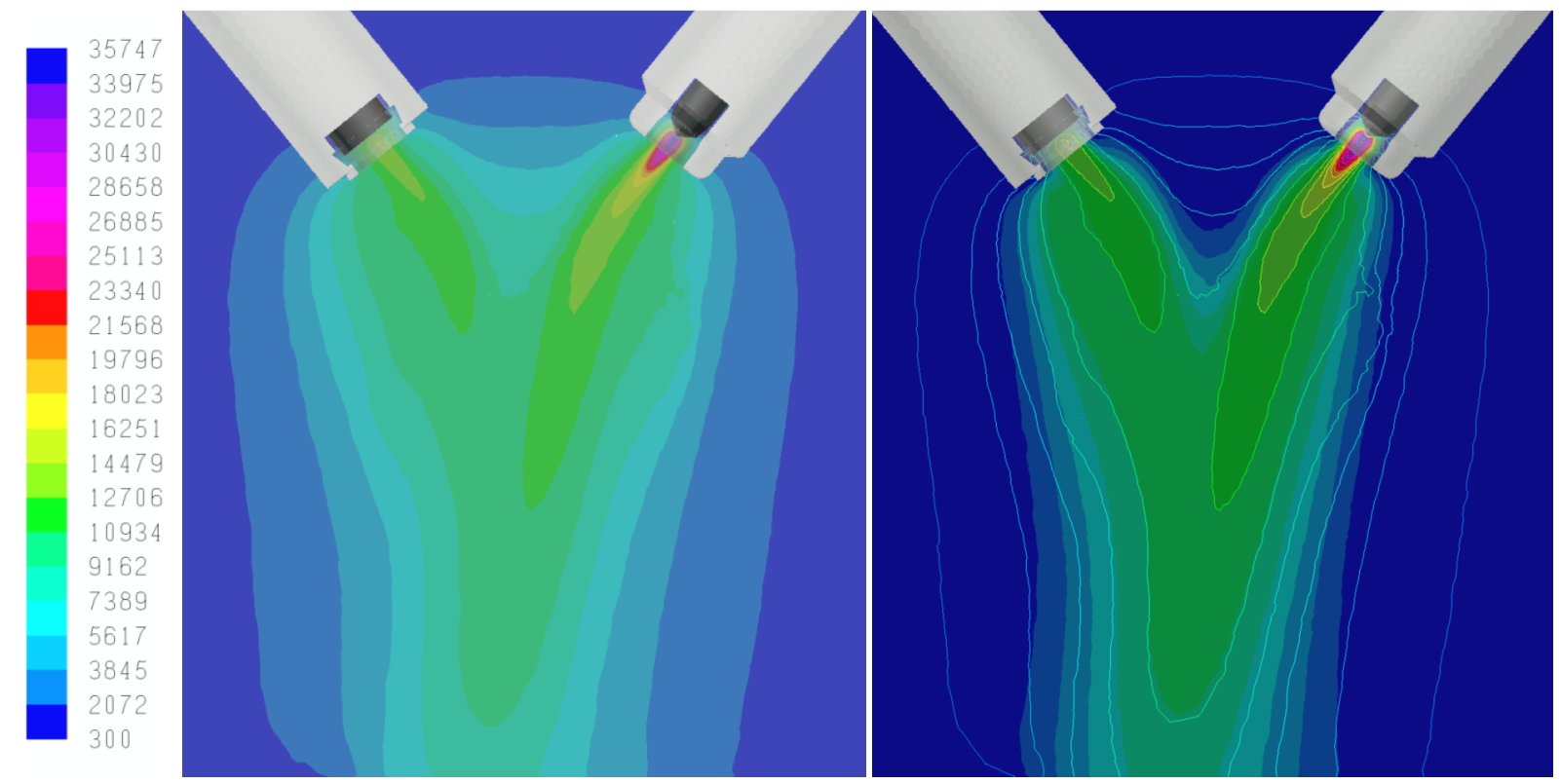

Figure 9. Front view of the electron (left) and heavy particles (right) temperature field $[\mathrm{K}]$ on the mid plane for the $2 \mathrm{~T}$ model. Electron temperature contour lines are superimposed on the heavy particles field contour.

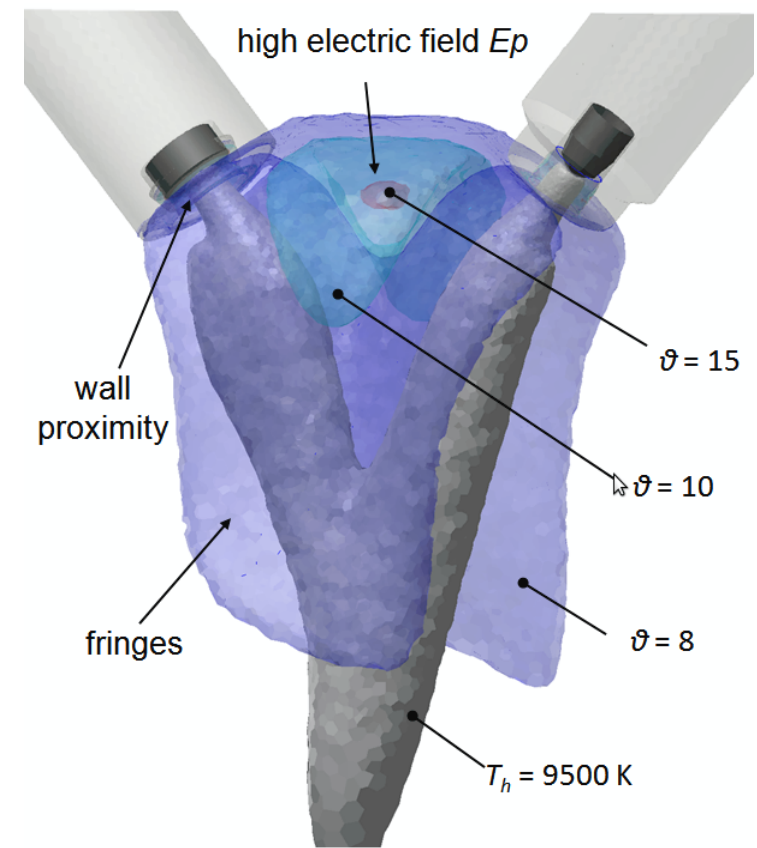

Figure 10. $\vartheta$ isosurfaces over the 9'500 K heavy particles temperature isosurface for the 2T model.

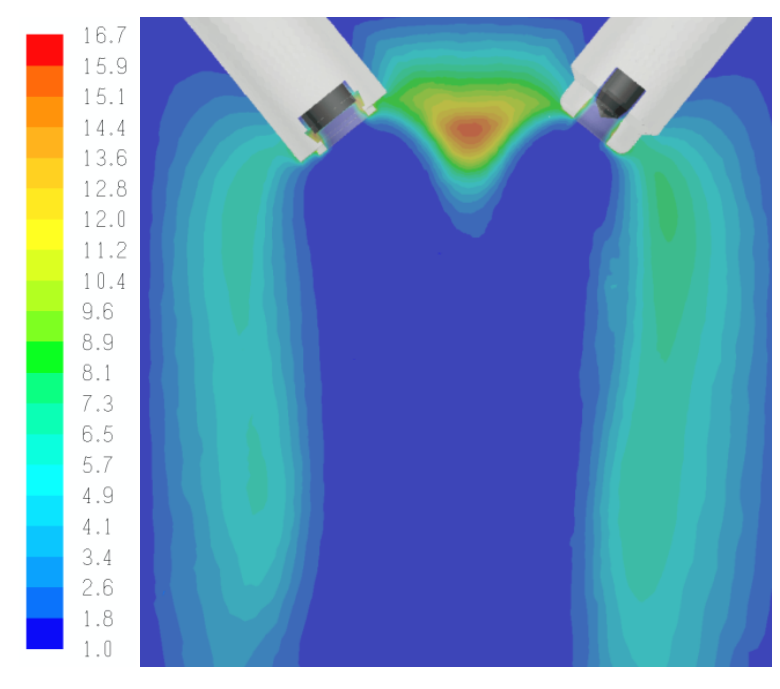

Figure 11. $\vartheta$ contour on the mid plane for the $2 \mathrm{~T}$ model. 


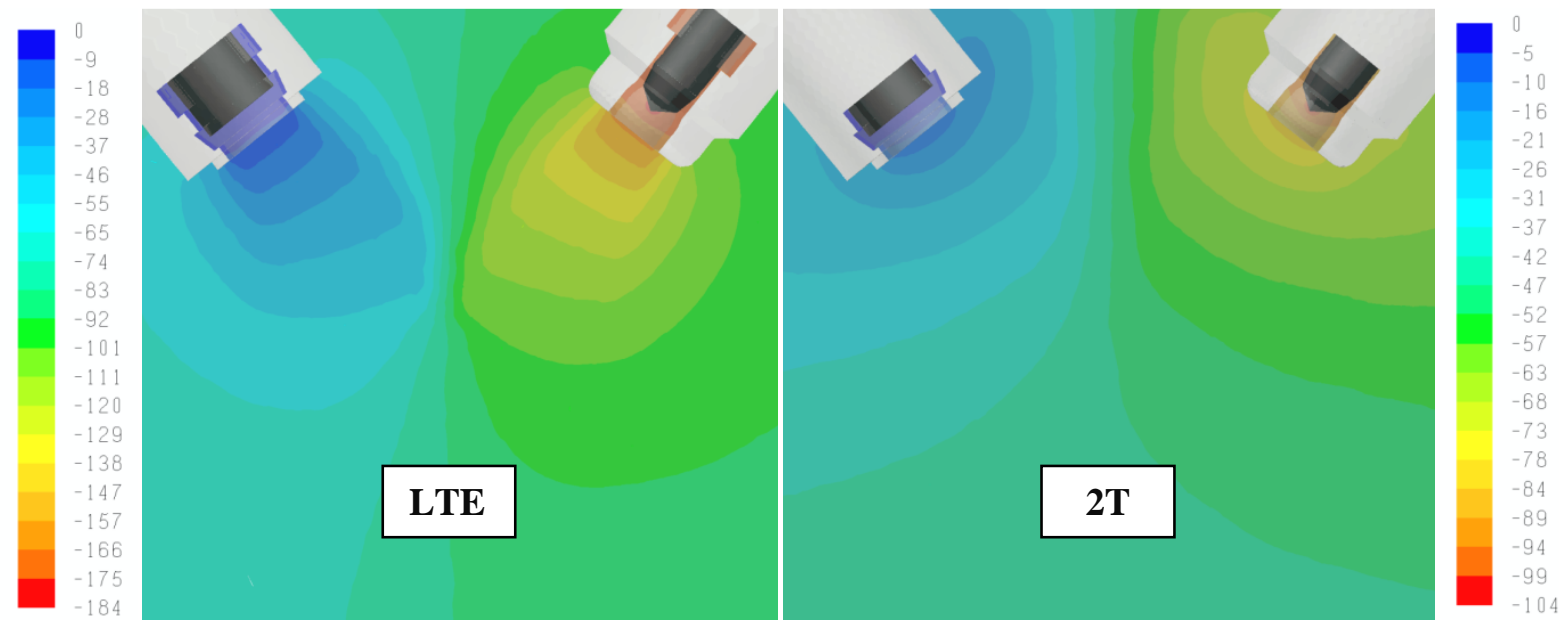

Figure 12. Electric potential field [V] for the LTE and 2T models. 


\section{References}

[1] Trelles J P, Chazelas C, Vardelle A and Heberlein J V R 2009 J. Therm. Spray Technol. 18728

[2] Heberlein J V H and Murphy A B 2008 J. Phys. D: Appl. Phys. 41053001

[3] Salvati F et al. 1991 Application of Plasma System for Tundish Heating Proc. of the 1st European Conference on Continuous Casting (Firenze, Italy)

[4] Salvati F. 2002 Development of Thermal Technologies for Pyrolysis and Combustion of Waste with Vitrification of Ash, Proc. of the 22nd Int. Conf. on Incineration and Thermal Treatment Technologies (Orlando, Florida, USA)

[5] Johnson T P, Deegan D E and Chapman C D 2001 Twin Plasma Torch Apparatus Tetronics Ltd Patent WO01/78471

[6] Barthelemy B., Girold C., Delalondre C., Paya B. and Baronnet J.M. 2003 Modeling a pilotscale combustion vitrification furnace under oxygen plasma arc transferred between twin torches, proceedings of the 16th Int. Symp. on Plasma Chemistry (ISPC-16), Taormina, Italy, 22-27 June 2003, Eds. R.D'Agostino, P.Favia, F.Fracassi, F.Palumbo (Dept. of Chemistry, University of Bari \& IMIP, CNR, Bari), paper n. 656

[7] Colombo V, Ghedini E, Mentrelli A and Malfa E 2005, 3-D Modelling of DC Transferred arc Twin Torch for Asbestos Inertization. In Nuclear Reactor Physics. A collection of papers dedicated to Silvio Edoardo Corno, 169-196, ISBN: 88-7992-211-4, Torino, C.L.U.T.

[8] Tang K M, Yan J D, Chapman C and Fang M T C 2010 J. Phys. D: Appl. Phys. 43345201

[9] Colombo V, Concetti A and Ghedini E 2008 IEEE Trans. Plasma Sci 361038

[10] Rat V, Murphy A B, Aubreton J, Elchinger M F and Fauchais P 2008 J. Phys. D: Appl. Phys. 41 183001

[11] Haidar J 1999 J. Phys. D: Appl. Phys. 32263

[12] Lowke J J, Morrow R and Haidar J 1997 J. Phys. D: Appl. Phys. 302033

[13] Colombo V, Ghedini E and Sanibondi P 2009 J. Phys. D: Appl. Phys. 42055213

[14] Cressault Y and Gleizes A 2004 J. Phys. D: Appl. Phys. 37560

[15] Hsu K C, Etemadi K and Pfender E 1983 J. Appl. Phys. 541293

[16] Henmi R, Yokomizu Y and Matsumura T 2004 The Journal of The Institute of Electrical Engineers of Japan 23988 